\title{
Consolidating APSIC: a professional and educational platform for the generation of young interventional cardiologists from the Asia-Pacific region
}

\author{
सहा \\ Ashok Seth*, FRCP, FESC, MSCAI, FAPSIC, DSc \\ President, Asian Pacific Society of Interventional Cardiology \\ Fortis Escorts Heart Institute, New Delhi, India
}

The last 18 months have been unprecedented in our lifetime. The COVID-19 pandemic has affected the entire world and has, at times, brought humanity and its activities to a standstill. It not only created new ways of living in the world but introduced previously unimagined virtual interactions. All this happened soon after I took over the Presidency of the Asian Pacific Society of Interventional Cardiology (APSIC) in November 2019. Despite the numerous limitations for in-person meetings, there was an opportunity to broaden the participation and the reach of the virtual meetings. And so, in April 2020, we started conducting virtual board meetings and bimonthly scientific sessions, leading to a virtual Asian Interventional Cardiovascular Therapeutics (AICT)AsiaPCR session in December 2020. The vicious second wave of the Delta virus, however, brought the worse form of contagion we had ever seen.

The rapidity and seriousness of the second wave of COVID19 overwhelmed our healthcare system. Hospitals overflowed with patients on oxygen and ventilators. It was both morally and physically exhausting; most families in India were affected, with close friends or relatives succumbing to COVID-19. This gave us no time to advance a number of ambitious plans that we had for APSIC this year. We worked hard to create a new format, a new edition of AICT-AsiaPCR 2021, with virtual sessions transmitted from three studios located in Singapore, New Delhi and Kuala Lumpur, and in-person attendance. This educational and interactive event, held 8-9 October 2021, was exciting, especially for the young generation of interventional cardiologists of the AsiaPacific region. This year we have also been able to "refresh" AsiaIntervention, the official journal of APSIC, with a new editorial board led by Prof. Upendra Kaul. This has led to the journal now being on track for possible PubMed indexation.

We have an ambitious agenda to complete over the next year as we emerge from the wrath of the COVID-19 pandemic and gradually start to normalise. In terms of virtual education, monthly virtual scientific symposia, primarily targeting a generation of young cardiologists, are planned. We need to encourage research and 
hence will create APSIC scholarships for worthy applicants who may want to spend time at other centres in the Asia-Pacific region for research or skill enhancement. Partnerships are planned with other interventional societies across the world, both for collaboration in "consensus statements", as well as for scientific sessions. Reconstituting the APSIC board to include representatives of various national interventional societies of the Asia-Pacific region will be an important step in cementing these relationships and working together. We also need to encourage and create greater opportunities for participation of women in interventional cardiology. Hence, we will create a subcommittee for this purpose and hope that we can work to overcome the gender inequality in interventional cardiology, where fewer than $5 \%$ are women. There is a lot of work to do over the next year, but I am sure we will be able to accomplish new dimensions by working together with focus and determination. We are cut out for the task before us, the vision is clear, and the time is right to strengthen APSIC as its mission is "Transforming lives through advancing innovation and global partnerships."

\section{Conflict of interest statement}

The author has no conflicts of interest to declare. 